\section{(2) OPEN ACCESS}

\title{
Contrasting contributions of TNF from distinct cellular sources in arthritis
}

\author{
Andrey Kruglov $\odot{ }^{1}, 2,3$ Marina Drutskaya, ${ }^{2}$ Dirk Schlienz, ${ }^{1}$ Ekaterina Gorshkova, ${ }^{2,3}$ \\ Katharina Kurz, ${ }^{1}$ Lars Morawietz, ${ }^{4}$ Sergei Nedospasov ${ }^{2,3,5}$
}

\begin{abstract}
Handling editor Josef S Smolen
\end{abstract}

- Additional material is published online only. To view please visit the journal online (http://dx.doi.org/10.1136/ annrheumdis-2019-216068).

${ }^{1}$ Chronic inflammation, Deutsches RheumaForschungszentrum, a Leibniz Institute, Berlin, Germany ${ }^{2}$ Center for Precision Genome Editing and Genetic Technologies for Biomedicine, Engelhardt Institute of Molecular Biology RAS, Moscow, Russian Federation ${ }^{3}$ Belozersky Institute of PhysicoChemical Biology and Biological Faculty, Lomonosov Moscow State University, Moscow,

Russia

${ }^{4}$ Institute of Pathology,

Medizinischen

Versorgungszentrum im

Fürstenberg-Karree Berlin, Berlin, Germany

Institute of Cell Biology and Neurobiology, Charité - Universitätsmedizin Berlin Berlin, Berlin, Germany

Correspondence to Dr Andrey Kruglov, Chronic inflammation, Deutsches Rheuma-Forschungszentrum Berlin, 10117 Berlin, Germany; kruglov@drfz.de

Professor Sergei Nedospasov; sergei.nedospasov@gmail.com

Received 23 July 2019 Revised 17 May 2020 Accepted 24 June 2020 Published Online First 12 August 2020

\section{Check for updates}

(C) Author(s) (or their employer(s)) 2020. Re-use permitted under CC BY-NC. No commercial re-use. See rights and permissions. Published by BMJ.

To cite: Kruglov A, Drutskaya M, Schlienz D, et al. Ann Rheum Dis 2020;79:1453-1459.

\section{ABSTRACT}

Objectives Neutralisation of tumour necrosis factor (TNF) is widely used as a therapy for rheumatoid arthritis (RA). However, this therapy is only effective in less than a half of patients and is associated with several side effects. We hypothesised that TNF may possess non-redundant protective and immunomodulatory functions in vivo that cannot be blocked without a cost. The present work aimed to identify cellular sources of protective and pathogenic TNF, and its molecular forms during autoimmune arthritis.

Methods Mice lacking TNF expression by distinct cell types, such as myeloid cells and T or B lymphocytes, were subjected to collagen-induced arthritis (CIA) and collagen antibody-induced arthritis. Mice lacking soluble TNF production were also employed. The severity and incidence of the disease, as well as humoral and cellular responses were assessed.

Results Myeloid cell-derived TNF contributes to both induction and pathogenesis of autoimmune arthritis. Conversely, T cell-derived TNF is protective during the induction phase of arthritis via limiting of interleukin-12 production by dendritic cells and by subsequent control of autoreactive memory T cell development, but is dispensable during the effector phase of arthritis. B cell-derived TNF mediates severity of CIA via control of pathogenic autoantibody production.

Conclusions Distinct TNF-producing cell types may modulate disease development through different mechanisms, suggesting that in arthritis TNF ablation from restricted cellular sources, such as myeloid cells, while preserving protective TNF functions from other cell types may be superior to pan-anti-TNF therapy.

\section{INTRODUCTION}

In humans, tumour necrosis factor (TNF) is overexpressed in several autoimmune diseases such as rheumatoid arthritis (RA), ankylosing spondylitis and others. ${ }^{1-3}$ Furthermore, TNF overexpression in mice leads to polyarthritis. ${ }^{4}$ Although other factors are also involved in arthritis pathogenesis, TNF inhibition as monotherapy is beneficial for a fraction of patients with RA and is widely used as treatment. ${ }^{3}$ The success of such therapy points to the contribution of non-redundant and deleterious functions of TNF in the development of RA. On the other hand, TNF has been implicated in multiple aspects of immune regulation. Specifically, TNF may control the development of CD4 $\mathrm{T}$ helper (Th) 1 and Th17 T cells in vivo ${ }^{5-7}$ and contributes to germinal centre (GC)-mediated antibody induction. ${ }^{9}$ Moreover, TNF induces expression of

\section{Key messages}

What is already known about this subject?

- Anti-tumour necrosis factor (TNF) therapy is widely used as a treatment for rheumatoid arthritis (RA) and is associated with several side effects that can be explained by beneficial functions of TNF that should not be neutralised.

\section{What does this study add?}

- The study links deleterious and protective TNF functions in arthritis to distinct cellular sources and molecular forms.

- TNF produced by myeloid cells controls arthritis induction through regulation of synovial fibroblast activation, while B cellderived TNF regulates severity via induction of autoantibodies.

- TNF produced by T cells limits arthritis development via control of T cell responses during induction phase of arthritis but is dispensable for the pathogenesis.

\section{How might this impact on clinical practice or future developments? \\ - Multifaceted contributions of TNF from distinct cellular sources at different stages of autoimmune arthritis suggest the advantage of cell type-specific TNF blockade for patients with RA and lay the ground for exploring myeloid cell-restricted TNF inhibition in arthritis.}

chemokines and adhesion molecules that mediate homing of proinflammatory cells. ${ }^{10} 11$ One of the first TNF-mediated events required for arthritis induction is the activation of pathogenic synovial fibroblast subsets, accompanied by enhanced matrix metalloproteases (MMPs) expression. ${ }^{12}$ Activation of MMPs within the joints further leads to tissue destruction and to antigen exposure that result in recruitment of inflammatory lymphocytes and amplification of inflammation. ${ }^{13} 14$ Studies of TNF inhibition in patients with RA indicate that TNF is involved in homing of proinflammatory cells, regulating the balance between $\mathrm{T}$ regulatory and $\mathrm{T}$ effector cells, as well as activating myeloid cells. ${ }^{1} 1516$ However, it remains unknown exactly which cellular sources of TNF may control these multiple effects during arthritis.

Here, we delineated the distinct contributions of TNF from selected cellular sources in two experimental arthritis models. First, we identified TNF 

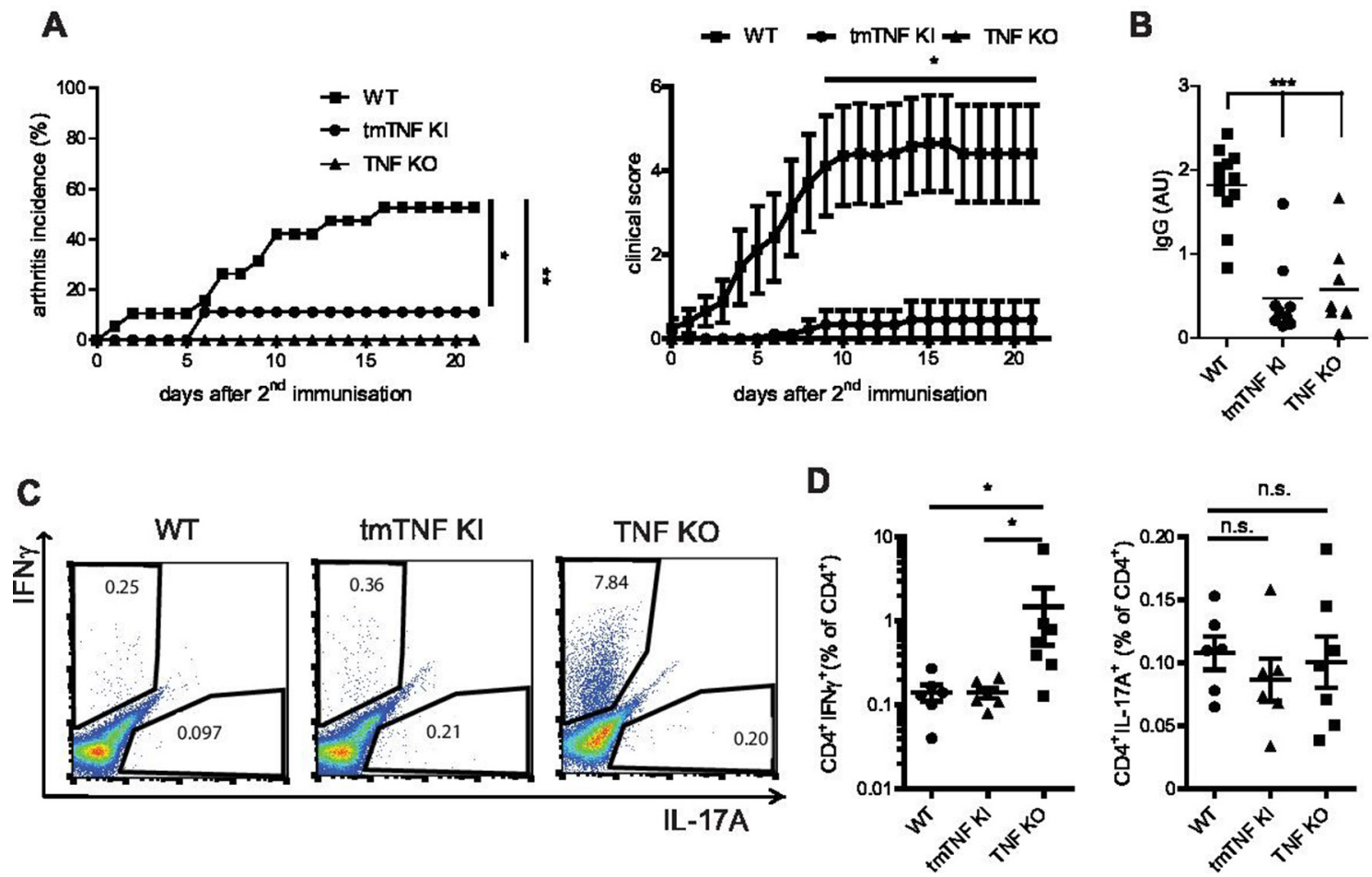

Figure 1 Soluble TNF is required for arthritis induction. (A) Incidence (left) and clinical scores (right) of CIA in tmTNF KI, WT and TNF KO mice. (B) Anti-collagen IgG titres in tmTNF KI, WT and TNF KO mice at day 14 after second immunisation. (C, D) Frequency of splenic Cll-specific Th1 (IFN $\gamma^{+}$) and Th17 (IL-17A $)$ CD4 T cell responses in tmTNF KI, WT and TNF KO mice at day 14 after second immunisation. All data are representative of two or more independent experiments with $n \geq 10$ for the analysis of arthritis incidence and severity and $n \geq 3$ for analysis of cellular and humoral responses. ${ }^{*} p<0.05,{ }^{* *} p<0.01,{ }^{* *} p<0.001$, as calculated by Kruskal-Wallis non-parametric test with Dunn's multiple comparisons test. CIA, collagen-induced arthritis; tmTNF, transmembrane form of TNF; TNF, tumour necrosis factor; WT, wild-type.

produced by myeloid cells as a critical factor for arthritis induction and pathogenesis. Second, we showed that T cell-derived TNF, likely in its membrane-bound form, provides protective functions through regulation of autoreactive T cell development during arthritis induction, but is dispensable during effector phase of arthritis. Finally, B cell-derived TNF controls development of autoantibodies during collagen-induced arthritis (CIA) and regulates severity of arthritis. Thus, our findings dissect the multifaceted contributions of TNF at different stages of autoimmune arthritis and underline intrinsic limitations of a nonselective pan-blockade of TNF.

\section{RESULTS}

\section{Soluble TNF is critical for CIA development}

TNF is a trimeric proinflammatory cytokine initially expressed in a membrane-bound form and then cleaved off the membrane by metalloproteases, such as ADAM17. ${ }^{17} 18$ Membrane-bound TNF is biologically active on cell-to-cell contacts and can also mediate reverse signalling. ${ }^{19-21}$ In order to address the contribution of soluble versus membrane-bound TNF in experimental arthritis, we first studied CIA in mice expressing only transmembrane form of TNF ( $\mathrm{tmTNF} \mathrm{KI})^{22}$ or in mice lacking TNF expression by all cells (TNF KO). ${ }^{23}$ Around 50\% of wild-type (WT) animals consistently developed CIA, ${ }^{24}$ while TNF-deficient and tmTNF $\mathrm{KI}$ mice failed to show any disease symptoms (figure $1 \mathrm{~A}$ and online supplementary table S1), indicating that soluble TNF is critical for disease induction.

Taking into account that both autoreactive cytokine-producing $\mathrm{T}$ cells and autoantibodies may contribute to arthritis development and disease severity, we next evaluated these parameters in mice lacking TNF entirely or only its soluble form. Anticollagen II
IgG titres were severely diminished in both TNF KO and tmTNF KI mice (figure 1B), indicating a crucial role of soluble TNF for autoantibody production. TNF has been reported to control both development and persistence (pathogenic memory) of autoreactive T cell responses. ${ }^{525}$ Given the importance of autoreactive memory $\mathrm{T}$ cells in disease perpetuation, we next quantified the frequency of collagen-specific CD4 T cells during active disease. Complete ablation of TNF resulted in augmented autoreactive CII-specific Th1 $\mathrm{T}$ cell responses characterised by increased IFN $\gamma$ when compared to WT or tmTNF KI mice (figure 1C and D). Notably, these cytokine-producing CD4 T cells were found to be antigen-specific (online supplementary figure S1). Altogether these data suggested that soluble TNF is critical for disease induction and autoantibody production, whereas membrane-bound TNF limits autoreactive T cell development.

Opposite contributions of TNF produced by myeloid cells and $\mathrm{T}$ cells in $\mathrm{CIA}$

TNF produced by various cell types may possess distinct nonredundant functions in various disease models. ${ }^{2627}$ Thus, we next compared the contribution of TNF expressed by myeloid cells and $\mathrm{T}$ cells in arthritis induction. Mice lacking TNF in myeloid cells (M-TNF KO) exhibited significantly decreased disease incidence (figure 2A, C and D and online supplementary table S1), indicating that TNF from myeloid cells is pathogenic. In contrast, mice with TNF ablation in T lymphocytes (T-TNF KO) were highly susceptible to arthritis development (figure $2 \mathrm{~B}-\mathrm{D}$ and online supplementary table S1), suggesting that T-TNF may actually play a protective immunomodulatory role in the same disease model. Furthermore, analysis of infiltration of myeloid cells (acute inflammation) and lymphocytes (chronic inflammation) in affected joints revealed 
A
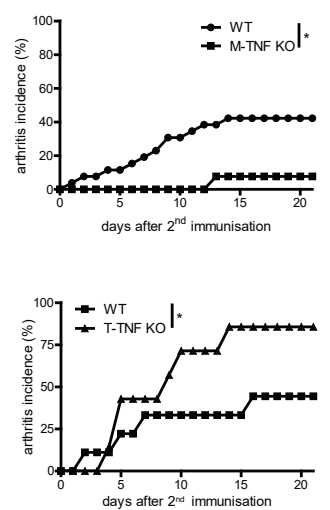

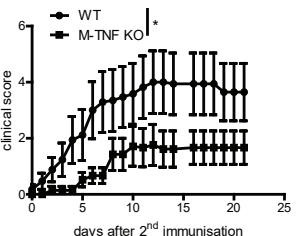

days after $2^{\text {nd }}$ immunisation

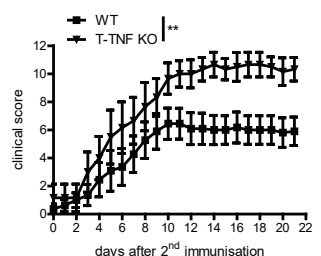

C
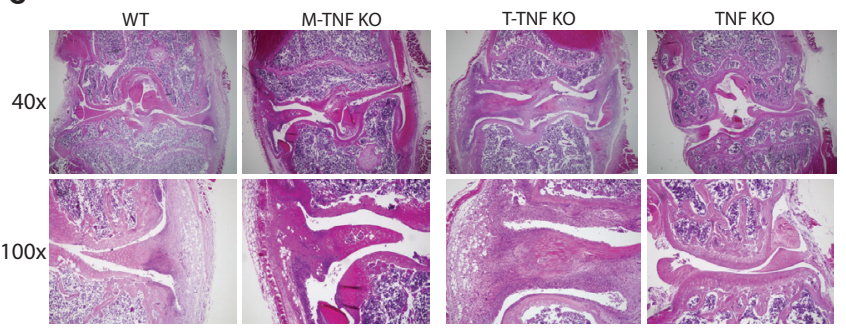

D
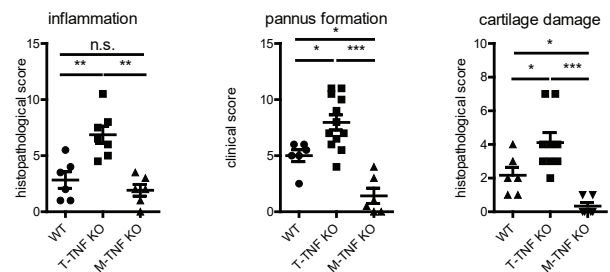

Figure 2 Cell type-specific requirements for TNF in CIA. Incidence (left) and clinical scores (right) of CIA in M-TNF KO (A), T-TNF KO (B) compared with WT mice. (C) H\&E staining of knee joints from TTNF KO, M-TNF KO, TNF KO and WT mice at day 14 after secondary immunisation. (D) Histopathological score of arthritis in T-TNF KO, MTNF KO, TNF KO and WT mice at day 14 after secondary immunisation. All data are representative of two or more independent experiments with $n \geq 10$ for the analysis of arthritis incidence and severity and $n \geq 3$ for analysis of cellular and humoral responses. ${ }^{*} p<0.05,{ }^{* *} p<0.01$, ${ }^{* * *} p<0.001$, as calculated by Kruskal-Wallis non-parametric test with Dunn's multiple comparisons test. CIA, collagen-induced arthritis; M-TNF, mice lacking TNF in myeloid cells; T-TNF, TNF ablation in T lymphocytes; TNF, tumour necrosis factor; WT, wild-type.

that T-TNF ablation was associated with increased CIA severity (figure 2D). Notably, deletion of myeloid cell-derived TNF did not affect accumulation of lymphoid cells, but led to decreased cartilage destruction (figure 2D), suggesting that M-TNF contributed to joint destruction during CIA. Thus, myeloid cell-derived and T cellderived TNF exhibited opposing effects both in arthritis induction and in the control of disease severity.

\section{Soluble TNF produced by myeloid cells is required for arthritis induction}

Given that TNF produced by myeloid cells, on one hand, and the soluble form of TNF, on the other hand, are essential contributors for RA development, we next tested whether soluble form of TNF from myeloid cells is critical for arthritis induction. To do so, we generated mice expressing only transmembrane TNF by myeloid

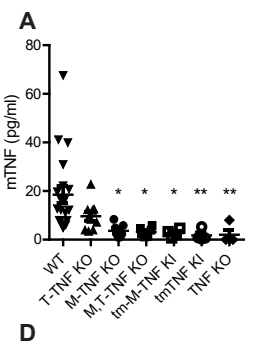

C
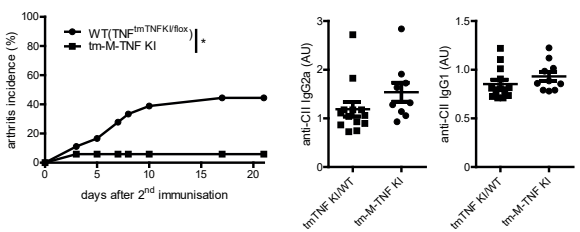

E $100 x$

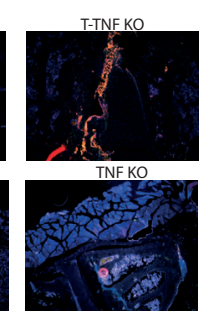

$\mathbf{F}$
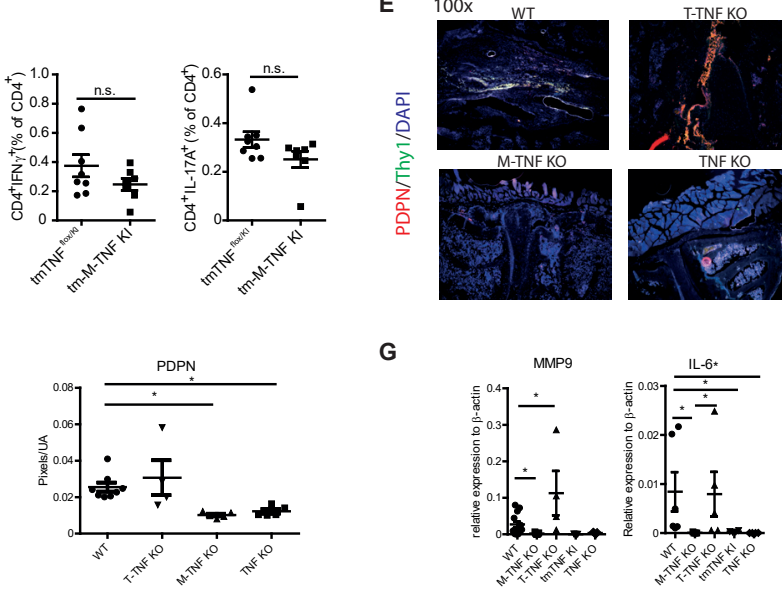

H

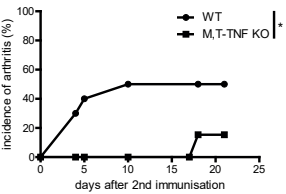

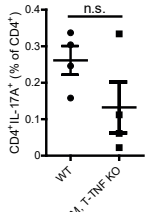

Figure 3 Critical role of soluble myeloid cell-derived TNF in arthritis induction. (A) TNF levels in sera of mice with cell type-specific TNF ablation and WT mice 1 day prior second immunisation. (B) Incidence of $\mathrm{CIA}$ in tm-M-TNF KI and WT mice. (C) Anti-collagen IgG titres in tm-M-TNF KI and WT mice at day 14 after second immunisation. (D) Frequency of anticollagen type II Th1 (IFN $\gamma^{+}$) and Th17 (IL-17A ${ }^{+}$) CD4 $\mathrm{T}$ cell responses in tm-M-TNF KI and WT mice at day 14 after second immunisation. (E, F) Immunofluorescent staining (THY1, gp38) of knee joints from WT, T-TNF KO, M-TNF KO and TNF KO at day 14 after arthritis induction. (G) IL-6 and MMP9 mRNA expression levels in synovial tissue of WT, M-TNF KO, T-TNF KO, tmTNF KI and TNF KO mice at day 21 after immunisation. (H) Incidence of CIA on M, T-TNF KO and WT mice. (I) Frequency of anticollagen type II Th1 (IFN $\left.\gamma^{+}\right)$and Th17 (IL-17A ${ }^{+}$) CD4 $\mathrm{T}$ cell responses in M,T-TNF KO and WT mice at day 14 after second immunisation. All data are representative of two or more independent experiments with $n \geq 10$ for the analysis of arthritis incidence and severity and $n \geq 3$ for analysis of cellular and humoral responses. ${ }^{*} \mathrm{p}<0.05,{ }^{* *} \mathrm{p}<0.01,{ }^{* *} \mathrm{p}<0.001$, as calculated by Kruskal-Wallis nonparametric test with Dunn's multiple comparisons test $(A)$, by Student's t-test (C, D, G). CIA, collagen-induced arthritis; M-TNF, mice lacking TNF in myeloid cells; tm-M-TNF, transmembrane TNF by myeloid cells; T-TNF, TNF ablation in T lymphocytes; TNF, tumour necrosis factor; WT, wildtype.

cells (tm-M-TNF KI mice) by crossing tmTNF KI mice to M-TNF $\mathrm{KO}$ animals. The resulting mice (tm-M-TNF KI) expressed only membrane-bound TNF on myeloid cells (online supplementary figure S2A,B), whereas both molecular forms of TNF were intact in all other cell types. Ablation of soluble TNF in myeloid cells resulted in decreased serum TNF levels during arthritis (figure 3A), whereas $\mathrm{T}$ cells did not contribute significantly to the systemic levels of TNF 
during CIA (figure 3A). Strikingly, ablation of soluble TNF production by myeloid cells resulted in reduced incidence of arthritis (figure $3 \mathrm{~B}$ and online supplementary table S1), while autoantibody responses and autoreactive $T$ cell responses were not affected (figure $3 \mathrm{C}$ and D). Thus, soluble TNF from myeloid cells controlled the arthritis development without affecting autoreactive $\mathrm{T}$ cell and humoral responses.

Recent reports have highlighted the importance of local joint macrophages in the pathogenesis of arthritis. ${ }^{28}$ Therefore, we next analysed the degree of TNF deletion in various myeloid subsets. M-TNF KO mice exhibited efficient TNF deletion in neutrophils, while TNF has been only partially deleted in inflammatory monocytes (online supplementary figure S2C, D, E). Interestingly, analysis of TNF production by cells infiltrating inflamed paws revealed that in the joints of M-TNF KO mice TNF is produced only by T cells, indicating that neither infiltrating, nor resident myeloid cells are the source of TNF in paws of arthritic M-TNF KO animals. Furthermore, joint swelling is associated with influx of neutrophils in M-TNF KO mice, while both neutrophils and inflammatory monocytes were detected in arthritic paws of WT and T-TNF KO animals (online supplementary figure S3). Thus, it is plausible that TNF from both resident and circulating myeloid cells might contribute to arthritis induction.

Enhanced production of TNF may result in activation of synovial fibroblasts characterised by upregulation of podoplanin (PDPN) and Thy $1,{ }^{29} 30$ as well as expression of metalloproteases and interleukin-6, leading to tissue destruction. ${ }^{12}$ Thus, we next addressed whether TNF produced by myeloid cells may regulate synovial fibroblast activation. Notably, TNF KO mice showed no signs of synovial fibroblast activation as revealed by Thy1/PDPN staining (figure 3E,Fand data not shown), while TNF ablation in myeloid cells resulted in reduced presence of activated fibroblasts during CIA. Consistently, expression levels of MMP9, TNF and IL-6 were significantly decreased in M-TNF KO joints when compared with WT counterparts (figure $3 \mathrm{G}$ and online supplementary figure S2). Altogether, these data suggested that M-TNF, likely in soluble form, may control arthritis induction via activation of synovial fibroblasts.

\section{Concomitant ablation of TNF in myeloid cells and T cells results in decreased CIA incidence}

Next, we tested that M-TNF may be both necessary and sufficient for disease induction, while $\mathrm{T}$ cell-derived TNF exhibits its protective role during arthritis. For this, we induced arthritis in mice lacking TNF production by both cell types (M,T-TNF KO) and found that disease development was significantly abrogated in M,T-TNF KO mice as compared with WT counterparts (figure 3Hand online supplementary table S1). Furthermore, M,T-TNF KO animals exhibited elevated levels of IFN $\gamma$-producing $\mathrm{T}$ cells in spleen (figure 3I), thus phenocopying CIA development in TNF KO. These findings further corroborated on the importance of myeloid cell-derived TNF for CIA induction, even in the absence of protective T-TNF.

\section{Membrane-bound TNF produced by T cells controls disease severity by limiting the expansion of autoreactive $T$ cells}

Since arthritis development is driven by arthritogenic Th1 and Th17 CD4 T cells, ${ }^{31}{ }^{32}$ we hypothesised that TNF from various cellular sources may have distinct effects on arthritogenic $\mathrm{T}$ cell populations. To get further insights into how TNF produced by $\mathrm{T}$ cells may control arthritis development, we analysed the arthritogenic, cytokine-producing $\mathrm{CD}^{+}{ }^{+} \mathrm{T}$ cells in various TNF-deficient mice during active disease. Importantly, T-TNF KO mice contained more autoreactive IFN $\gamma$-producing and IL-17A-producing $\mathrm{CD}^{+}$ $\mathrm{T}$ cells in their spleens when compared with WT counterparts (figure 4A and B). Of note, myeloid cell-derived TNF did not
A live cells from inflammed paws

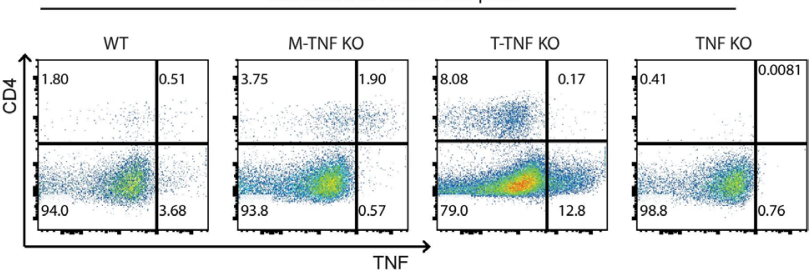

WT

M-TNF KO T-TNF KO

TNF KO

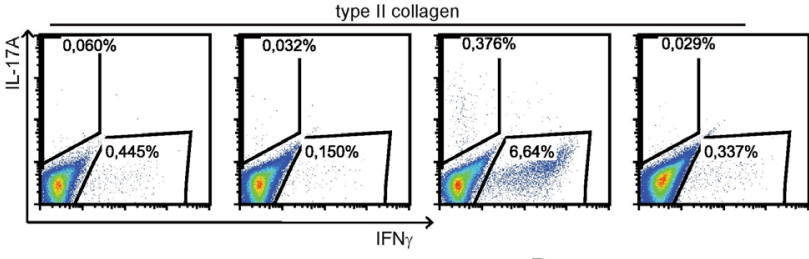

C

type II collagen

D
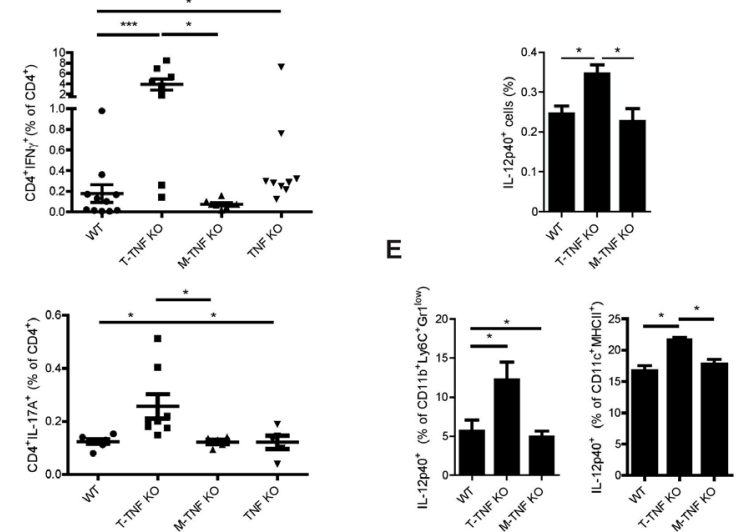

Figure 4 T-TNF regulates memory T cell responses during CIA. (A) Analysis of TNF production in inflamed paws in TNF KO, T-TNF KO, M-TNF KO and WT mice at day 14 after second immunisation. $(B, C)$ Frequency of anticollagen type II Th1 (IFN $\gamma^{+}$) and Th17 (IL-17A ${ }^{+}$) CD4 T cell responses in T-TNF KO, M-TNF KO and WT mice in spleen at day 14 after second immunisation. Frequency of total IL-12p40-producing cells

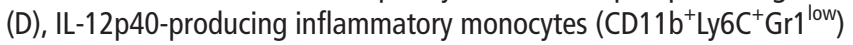
and $\left(\mathrm{CD} 11 \mathrm{c}^{+} \mathrm{MHCII}^{+}\right)(\mathrm{E})$ in spleens of WT, T-TNF KO and M-TNF KO mice at day 6 after immunisation. Splenocytes were stimulated with heatkilled M.TB H37Ra (100 mcg/mL) for 6 hours in the presence of brefeldin $A$ and were analysed for the IL-12p40 expression using flow cytometry. All data are representative of two or more independent experiments with $n \geq 3$. ${ }^{*} p<0.05,{ }^{* *} p<0.01,{ }^{* * *} p<0.001$, as calculated by KruskalWallis non-parametric test with Dunn's multiple comparisons test. CIA, collagen-induced arthritis; M-TNF, mice lacking TNF in myeloid cells; T-TNF, TNF ablation in T lymphocytes; TNF, tumour necrosis factor; WT, wild-type.

influence frequencies of CII-specific Th1 or Th17 CD4 T cells (figure 4A and B). TNF-TNFR2 axis is known to modulate Treg numbers and function via control of FoxP3 expression ${ }^{33-35}$ and drive pathologic state of sinovial fibroblasts (SFs). ${ }^{36}$ However, ablation of TNF only in T cells or in myeloid cells had no impact neither on Treg numbers in lymphoid organs, nor on FoxP3 expression levels (online supplementary figure S4A, B), suggesting that T-TNF is dispensable for Tregs modulation in this model. Finally, blockade of IFN $\gamma$, but not of TNFR2 or IL-17A, significantly reduced arthritis incidence in those mice during CIA (online supplementary figure S4C and data not shown). Altogether, this suggests that TNF derived from T cells controls CIA development by limiting autoreactive Th1 response. 
Since TNF is known to control proinflammatory cytokine production by myeloid cells, ${ }^{5}$ we next quantified the number of IL-12p40-producing cells at day 6 after immunisation. Ablation of T cell-derived TNF, but not of TNF from myeloid cells, augmented frequencies of IL-12p40-producing cells in the spleen (figure 4C), suggesting that in WT mice T-TNF may dampen IL-12p40 production in vivo during immune responses. Further analysis revealed increased numbers of IL-12p40-producing monocytes and dendritic cells on T-TNF deletion (figure 4D), while the frequency of inflammatory monocytes was not changed in these mice (online supplementary figure S4D, E). Altogether, these data suggest that T-TNF regulates Th1 and Th17 responses via control of IL-12p40 production by inflammatory monocytes and dendritic cells.

\section{Myeloid cell-derived TNF drives pathogenesis in collagen- antibody induced arthritis}

Cell type-specific TNF deletion strategy during CIA affected gene expression both during induction of autoimmune responses and during effector phase of autoimmune disease. In order to further delineate the contribution of distinct cellular sources to arthritis pathogenesis, we utilised the model of collagen-antibody induced arthritis (CAIA) that is driven by preformed autoantibodies, is independent on the adaptive immune system and is known to be critically dependent on soluble TNF. ${ }^{37}$ TNF KO mice were largely protected from the disease development (figure 5A). Strikingly, myeloid cell-derived TNF was found to be crucial for CAIA development (figure 5A-C), while T-TNF was dispensable for disease manifestation (figure 5A-C). Furthermore, both TNF KO and M-TNF KO mice exhibited reduced activation of synovial fibroblasts during CAIA (figure 5D), when compared with WT mice. Of note, gp $38^{+}$Thy $1^{-}$SFs that are important for the tissue destruction seemed to be absent in mice lacking TNF in myeloid cells on CAIA (figure 5D). Finally, M-TNF KO mice had significantly lower IL-6 levels in their blood during CAIA (figure 5E). Altogether, myeloid cell-derived TNF drives arthritis induction during CAIA via activation of synovial fibroblasts and IL-6 production.

\section{B cell-derived TNF controls autoantibody production and disease severity during $\mathrm{CIA}$}

Since TNF controls autoantibody development during CIA (figure 1), we next wondered which cellular source is crucial for anti-CII antibody production during CIA. Several studies have described the critical contribution of $\mathrm{B}$ cell-derived TNF for the follicular dendritic cell development and GC reactions. ${ }^{8} 938$ Thus, we next analysed anti-CII IgG titres (figure 6A) in various knockout strains during CIA and observed the reduction of anticollagen type II-specific IgG, IgG1 and IgG2a titres in B-TNF $\mathrm{KO}$ mice during CIA (figure 6A). Interestingly, B-TNF KO developed disease with similar incidence when compared to WT littermate controls (figure 6B), but with significantly lower clinical score (figure 6C), suggesting that B-TNF controls severity of CIA. Furthermore, B cell-derived TNF controlled frequency of GC B cells in spleen during CIA, but did not influence numbers of either IFN $\gamma$-producing or IL-17A-producing CD4 T cells (figure 6D).

\section{DISCUSSION}

There are multiple biological therapies currently approved for RA treatment with predominance of anti-TNF biologics. ${ }^{12}$ However, anti-TNF therapy is associated with multiple, although relatively rare, side effects: susceptibility to intracellular
A

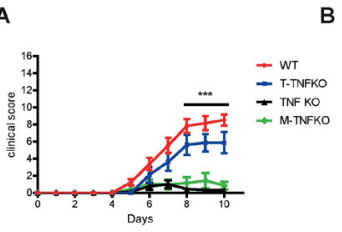

C
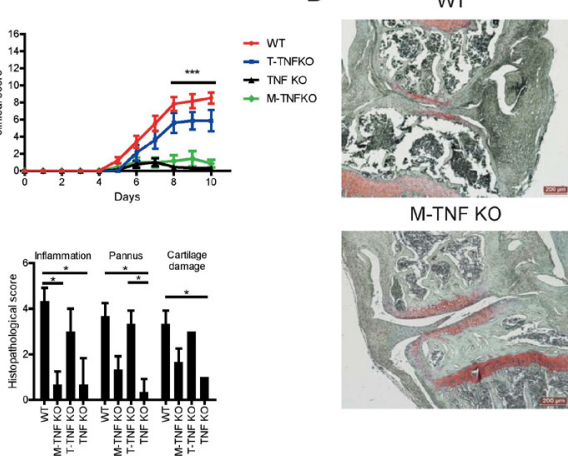

M-TNF KO

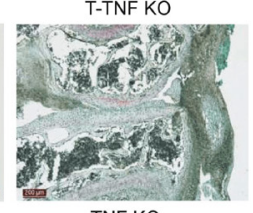

TNF KO
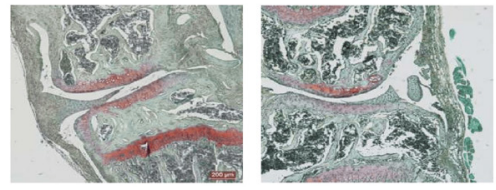

D naive
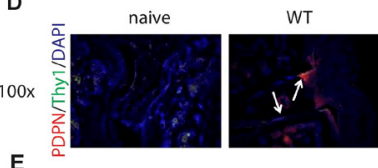

T-TNF KO
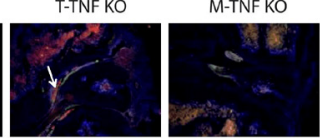

TNF KO

E

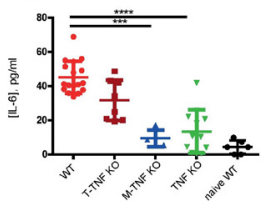

Figure 5 Myeloid-derived TNF controls severity of CAIA. (A) Clinical score of CAIA in M-TNF KO, T-TNF KO, TNF KO and WT mice. (B) Safranin 0 , fast green and haematoxylin staining of knee joints from T-TNF KO, M-TNF KO, TNF KO and WT mice at day 10 after induction of CAIA. (C) Histopathological scores of knee joint sections from T-TNF KO, M-TNF KO, TNF KO and WT mice at day 10 after arthritis induction. (D) Immunofluorescent staining (THY1, gp38) of knee joints from WT, T-TNF KO, M-TNF KO and TNF KO at day 10 after induction of CAIA. (E) Serum levels of IL-6 in WT, T-TNF KO, M-TNF KO and TNF KO at day 10 after induction of CAIA. All data are representative of two or more independent experiments with $n \geq 6$ for the analysis of arthritis clinical scores and serum IL-6 levels and $n \geq 3$ for histopathological evaluation. ${ }^{* *} p<0.05,{ }^{* *} p<0.01,{ }^{* *} p<0.001$, as calculated by Kruskal-Wallis nonparametric test with Dunn's multiple comparisons test. CAIA, collagen antibody-induced arthritis; M-TNF, mice lacking TNF in myeloid cells; T-TNF, TNF ablation in T lymphocytes; CIA, collagen-induced arthritis; WT, wild-type.

infections, particularly reactivation of Mycobacterium tuberculosis, secondary autoimmune manifestations and an increased risk of lymphoma development, all due to several known nonredundant beneficial functions of TNF in host defence, immune and body homeostasis. ${ }^{13940}$ Here we uncover the significance of non-redundant contributions of TNF from distinct cellular sources in the pathogenesis of experimental arthritis that implicate cell type-restricted TNF blockade as a promising strategy for the treatment of RA. Our data suggest that TNF produced by $\mathrm{T}$ cells controls activation of $\mathrm{T}$ cells in the secondary lymphoid organs and that T-TNF blockade using conventional anti-TNF therapy may abolish this protective effect, resulting in activation of autoreactive T cells capable of initiating secondary autoimmunity in patients treated with anti-TNF therapeutics.

Myeloid cells, a component of innate immune system, are long implicated in arthritis pathogenesis. ${ }^{41}$ Selective depletion of myeloid cell subsets, such as monocytes, macrophages and neutrophils, further revealed their critical impact on disease induction and severity. ${ }^{42} 43$ Using highly selective genetic TNF ablation in mice, we have demonstrated the functional importance of TNF produced by myeloid cells in disease pathogenesis. Mechanistically, M-TNF acts in its soluble form to activate 


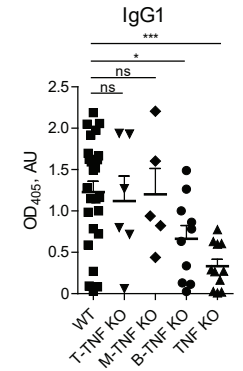

C

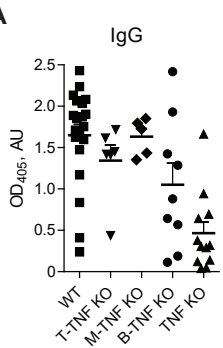

B

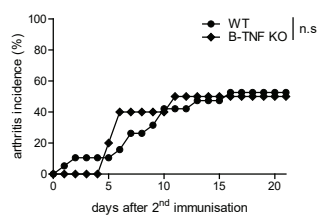

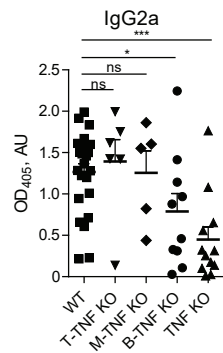

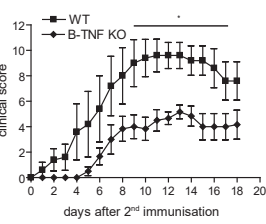

D

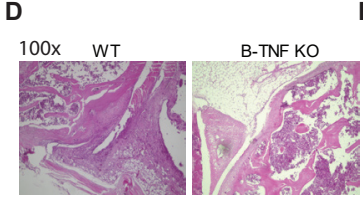

$\mathbf{F}$

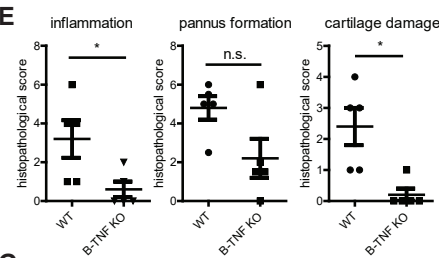

G

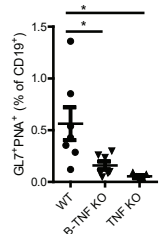

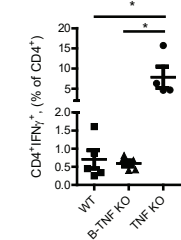

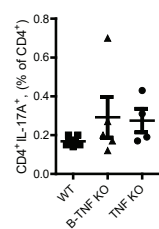

Figure 6 B cell-derived TNF controls severity of CIA. (A) Anticollagen type II antibody titres in WT, T-TNF KO, M-TNF KO, B-TNF KO and TNF KO mice at day 21 after immunisation. (B) Incidence of $\mathrm{CIA}$ in B-TNF KO, TNF KO and WT mice. (C) Clinical scores of CIA in B-TNF KO and WT mice. (D) Frequency of B cells (CD1 $9^{+}$cells) and GC B cells $\left(C D 19^{+} \mathrm{GL7}^{+} \mathrm{PNA}{ }^{+}\right)$in WT, B-TNF KO and TNF KO mice. (E) Frequency of anticollagen type II Th1 (IFN $\gamma^{+}$) and Th17 (IL-17A ${ }^{+}$) CD4 T cell responses in WT, B-TNF KO and TNF KO mice at day 14 after second immunisation. All data are representative of two or more independent experiments with $n \geq 10$ for the analysis of arthritis incidence and severity and $n \geq 3$ for analysis of cellular and humoral responses. ${ }^{*} p<0.05,{ }^{* *} p<0.01$, ${ }^{* * *} p<0.001$, as calculated by Kruskal-Wallis non-parametric test with Dunn's multiple comparisons test. GC, CIA, collagen-induced arthritis; GC, germinal centre; M-TNF, mice lacking TNF in myeloid cells; T-TNF, TNF ablation in T lymphocytes; TNF, TNF, tumour necrosis factor; WT, wild-type.

synovial fibroblasts. On the other hand, myeloid cells are the main contributors to systemic serum TNF in arthritic mice, suggesting that increased TNF levels may initiate arthritis in the presence of arthritogenic adaptive immunity. Indeed, M,T-TNF KO mice that lack TNF from myeloid cells and T cells displayed strongly reduced arthritis induction despite enhanced autoreactive cytokine-producing CD4 T cells and autoantibody development. TNFR signalling in synovial fibroblasts is crucial for synovial fibroblasts activation and may serve as the initial signal for arthritis development. ${ }^{123036}$ Altogether, it is plausible that soluble TNF produced by myeloid cells initiates arthritis via activation of fibroblasts in the joint in the presence of autoantibodies. Recent report described the development of bispecific TNF inhibitor that selectively inhibits TNF production by F4/80-expressing myeloid cells, ${ }^{44}$ and is intended for arthritis treatment through selective ablation of TNF on macrophages.

TNF exerts its biological actions via two distinct receptors, TNFR1 and TNFR2 with distinct functions: while signalling via TNFR1 is driving arthritis pathogenesis, ${ }^{12}$ 45-47 TNFR2 signalling exhibits protective functions. ${ }^{6} 33354647$ Membrane-bound TNF was reported to preferentially bind to TNFR2, ${ }^{48}$ and it is possible that the tmTNF-TNFR2 signalling axis is crucial for antigen presenting cells tuning by $\mathrm{T}$ cells in the secondary lymphoid organs. Indeed, blockade of both TNFR1 and TNFR2, but not TNFR1, not only ameliorated the disease, but also enhanced T cell responses. ${ }^{47}$ Finally, TNFR2 expressed on Tregs is instrumental for their suppressive action. ${ }^{33} 35$ Altogether, this data suggest that novel anti-TNF therapies should be designed not to interfere with tmTNF-TNFR2 axis to avoid enhanced development of autoimmune $\mathrm{T}$ cells. Finally, an earlier study revealed that $\mathrm{T}$ cell-derived TNF is critical for the control of chronic experimental M. tuberculosis infection, whereas myeloid cell-derived TNF is dispensable for the survival on M. tuberculosis challenge. ${ }^{27}$

Based on these findings we propose that the next generation anti-TNF therapy should preserve TNF produced by T cells, and that myeloid cell TNF constitutes reasonable selective therapeutic target for the treatment of arthritis. ${ }^{49}$

\section{MATERIALS AND METHODS}

Detailed explanation of each procedure was described in the online supplementary file 1 .

Mice with ablation of TNF in various cell types used in this study were described elsewhere. ${ }^{22} 26$ All animal procedures were carried out in accordance with German and Russian regulations for animal protection. CIA was performed by immunisation of mice with chicken collagen II in complete Freund's adjuvant. CAIA was induced by injection of monoclonal anti-CII antibodies (Chondrex). Histological analysis of knee joints was performed during CIA and CAIA. Analysis of cytokine production by $\mathrm{T}$ cells during CIA was performed by flow cytometry after restimulation with collagen II. Cytokine production and autoantibody production were assessed by ELISA. Gene expression was measured by real-time PCR. All results were statistically evaluated using by Kruskal-Wallis non-parametric test with Dunn's multiple comparisons test unless otherwise stated. P values $(p<0.05)$ were considered as statistically significant.

\section{Twitter Andrey Kruglov @AndreyKruglov6}

Acknowledgements We thank S Prepens and R Zvartsev for their help during this project; H Schäfer, S Gruczek and M Ohde for excellent animal husbandry; L Drutskaya and R Zvartsev for mouse genotyping; members of the German Rheumatism Research Center Flow Cytometry Core Facility (T Kaiser, J Kirsch) for help with FACS analysis and H Hecker-Kia, H Schliemann, T Geske and A Peddinghaus for preparation of antibodies. We thank Dr S Grivennikov (FCCC, USA) for his critical comments on the manuscript.

Contributors AK and SN designed research. AK, MD, DS, KK, EG and LM performed experiments. AK, MD and SN wrote the manuscript.

Funding This study was supported by Deutsches Forschungsgemeinschaft (NE1466/2-1; TRR241 A04), by Leibniz ScienceCampus Chronic Inflammation (www. chronische-entzuendung.org) and the Russian Science Foundation (grant 19-7530032 for CAIA experiments and 17-74-20059 for antibody responses). Genotyping of all the mouse lines was carried out with support from grant 075-15-2019-1660 from the Ministry of Science and Higher Education of the Russian Federation.

Competing interests None declared.

Patient and public involvement Patients and/or the public were not involved in the design, or conduct, or reporting or dissemination plans of this research.

Patient consent for publication Not required. 
Ethics approval All animal procedures were carried out in accordance with German and Russian regulations for animal protection.

Provenance and peer review Not commissioned; externally peer reviewed.

Data availability statement Data are available in a public, open access repository. All the data relevant to the study are included in the article or uploaded as supplementary information.

Open access This is an open access article distributed in accordance with the Creative Commons Attribution Non Commercial (CC BY-NC 4.0) license, which permits others to distribute, remix, adapt, build upon this work non-commercially, and license their derivative works on different terms, provided the original work is properly cited, appropriate credit is given, any changes made indicated, and the use is non-commercial. See: http://creativecommons.org/licenses/by-nc/4.0/.

ORCID iD

Andrey Kruglov http://orcid.org/0000-0002-4597-2087

\section{REFERENCES}

1 Tracey D, Klareskog L, Sasso EH, et al. Tumor necrosis factor antagonist mechanisms of action: a comprehensive review. Pharmacol Ther 2008;117:244-79.

2 Feldmann M, Williams RO, Paleolog E. What have we learnt from targeted anti-TNF therapy? Ann Rheum Dis 2010;69 Suppl 1:i97-9.

3 Taylor PC, Feldmann M. Anti-TNF biologic agents: still the therapy of choice for rheumatoid arthritis. Nat Rev Rheumatol 2009;5:578-82.

4 Keffer J, Probert L, Cazlaris H, et al. Transgenic mice expressing human tumour necrosis factor: a predictive genetic model of arthritis. Embo J 1991;10:4025-31.

5 Kruglov AA, Lampropoulou V, Fillatreau S, et al. Pathogenic and protective functions of TNF in neuroinflammation are defined by its expression in T lymphocytes and myeloid cells. J Immunol 2011;187:5660-70.

6 Notley CA, Inglis JJ, Alzabin S, et al. Blockade of tumor necrosis factor in collageninduced arthritis reveals a novel immunoregulatory pathway for Th1 and Th17 cells. J Exp Med 2008;205:2491-7.

7 Zakharova M, Ziegler HK. Paradoxical anti-inflammatory actions of TNF-alpha: inhibition of IL-12 and IL-23 via TNF receptor 1 in macrophages and dendritic cells. J Immunol 2005:175:5024-33.

8 Endres R, Alimzhanov MB, Plitz T, et al. Mature follicular dendritic cell networks depend on expression of lymphotoxin beta receptor by radioresistant stromal cells and of lymphotoxin beta and tumor necrosis factor by B cells. J Exp Med 1999:189:159-68.

9 Tumanov AV, Grivennikov SI, Kruglov AA, et al. Cellular source and molecular form of TNF specify its distinct functions in organization of secondary lymphoid organs. Blood 2010;116:3456-64.

10 Ngo VN, Korner H, Gunn MD, et al. Lymphotoxin alpha/beta and tumor necrosis factor are required for stromal cell expression of homing chemokines in B and T cell areas of the spleen. J Exp Med 1999;189:403-12.

11 Finsterbusch M, Voisin M-B, Beyrau M, et al. Neutrophils recruited by chemoattractants in vivo induce microvascular plasma protein leakage through secretion of TNF. J Exp Med 2014;211:1307-14.

12 Armaka M, Apostolaki M, Jacques P, et al. Mesenchymal cell targeting by TNF as a common pathogenic principle in chronic inflammatory joint and intestinal diseases. $J$ Exp Med 2008;205:331-7.

13 Burrage PS, Mix KS, Brinckerhoff CE. Matrix metalloproteinases: role in arthritis. Front Biosci 2006;11:529-43.

14 Joronen $\mathrm{K}$, Ala-aho R, Majuri M-L, et al. Adenovirus mediated intra-articular expression of collagenase-3 (MMP-13) induces inflammatory arthritis in mice. Ann Rheum Dis 2004;63:656-64.

15 Huang Z, Yang B, Shi Y, et al. Anti-TNF- $\alpha$ therapy improves Treg and suppresses Teff in patients with rheumatoid arthritis. Cell Immunol 2012;279:25-9.

16 Nadkarni S, Mauri C, Ehrenstein MR. Anti-TNF-alpha therapy induces a distinct regulatory $\mathrm{T}$ cell population in patients with rheumatoid arthritis via TGF-beta. J Exp Med 2007;204:33-9.

17 Black RA, Rauch CT, Kozlosky CJ, et al. A metalloproteinase disintegrin that releases tumour-necrosis factor-alpha from cells. Nature 1997:385:729-33.

18 Moss ML, Jin SL, Milla ME, et al. Cloning of a disintegrin metalloproteinase that processes precursor tumour-necrosis factor-alpha. Nature 1997;385:733-6.

19 Kriegler M, Perez C, DeFay K, et al. A novel form of TNF/cachectin is a cell surface cytotoxic transmembrane protein: ramifications for the complex physiology of TNF. Cell 1988:53:45-53.

20 Georgopoulos S, Plows D, Kollias G. Transmembrane TNF is sufficient to induce localized tissue toxicity and chronic inflammatory arthritis in transgenic mice. J Inflamm 1996;46:86-97.

21 Davignon J-L, Rauwel B, Degboé Y, et al. Modulation of T-cell responses by antitumor necrosis factor treatments in rheumatoid arthritis: a review. Arthritis Res Ther 2018:20:229

22 Ruuls SR, Hoek RM, Ngo VN, et al. Membrane-bound TNF supports secondary lymphoid organ structure but is subservient to secreted TNF in driving autoimmune inflammation. Immunity 2001;15:533-43.
23 Kuprash DV, Tumanov AV, Liepinsh DJ, et al. Novel tumor necrosis factor-knockout mice that lack Peyer's patches. Eur J Immunol 2005;35:1592-600.

24 Inglis JJ, Simelyte E, McCann FE, et al. Protocol for the induction of arthritis in C57BL/6 mice. Nat Protoc 2008:3:612-8.

25 Kassiotis G, Kollias $G$. Uncoupling the proinflammatory from the immunosuppressive properties of tumor necrosis factor (TNF) at the p55 TNF receptor level: implications for pathogenesis and therapy of autoimmune demyelination. J Exp Med 2001;193:427-34.

26 Grivennikov SI, Tumanov AV, Liepinsh DJ, et al. Distinct and nonredundant in vivo functions of TNF produced by T cells and macrophages/neutrophils: protective and deleterious effects. Immunity 2005;22:93-104.

27 Allie N, Grivennikov SI, Keeton R, et al. Prominent role for T cell-derived tumour necrosis factor for sustained control of Mycobacterium tuberculosis infection. Sci Rep 2013;3:1809.

28 Culemann S, Grüneboom A, Nicolás-Ávila José Ángel, et al. Locally renewing resident synovial macrophages provide a protective barrier for the joint. Nature 2019;572:670-5.

29 Croft AP, Campos J, Jansen $K$, et al. Distinct fibroblast subsets drive inflammation and damage in arthritis. Nature 2019:570:246-51.

30 Zhang F, Wei K, Slowikowski K, et al. Defining inflammatory cell states in rheumatoid arthritis joint synovial tissues by integrating single-cell transcriptomics and mass cytometry. Nat Immunol 2019;20:928-42.

31 Butler DM, Malfait AM, Maini RN, et al. Anti-IL-12 and anti-TNF antibodies synergistically suppress the progression of murine collagen-induced arthritis. Eur $J$ Immunol 1999:29:2205-12.

32 Malfait AM, Butler DM, Presky DH, et al. Blockade of IL-12 during the induction of collagen-induced arthritis (CIA) markedly attenuates the severity of the arthritis. Clin Exp Immunol 1998;111:377-83.

33 Chen X, Bäumel M, Männel DN, et al. Interaction of TNF with TNF receptor type 2 promotes expansion and function of mouse CD4+CD25+T regulatory cells. $J$ Immunol 2007;179:154-61.

34 Tsakiri N, Papadopoulos D, Denis MC, et al. Tnfr2 on non-haematopoietic cells is required for Foxp3+ Treg-cell function and disease suppression in EAE. Eur I Immunol 2012:42:403-12.

35 Atretkhany K-SN, Mufazalov IA, Dunst J, et al. Intrinsic TNFR2 signaling in T regulatory cells provides protection in CNS autoimmunity. Proc Natl Acad Sci U S A 2018;115:13051-6.

36 Sakkou M, Chouvardas P, Ntari L, et al. Mesenchymal TNFR2 promotes the development of polyarthritis and comorbid heart valve stenosis. JCI Insight 2018;3. doi:10.1172/jici.insight.98864. [Epub ahead of print: 05 Apr 2018].

37 Zalevsky J, Secher T, Ezhevsky SA, et al. Dominant-negative inhibitors of soluble TNF attenuate experimental arthritis without suppressing innate immunity to infection. $J$ Immunol 2007;179:1872-83.

38 Anolik JH, Ravikumar R, Barnard J, et al. Cutting edge: anti-tumor necrosis factor therapy in rheumatoid arthritis inhibits memory B lymphocytes via effects on lymphoid germinal centers and follicular dendritic cell networks. I Immunol 2008:180:688-92.

39 Lorenzetti R, Zullo A, Ridola L, et al. Higher risk of tuberculosis reactivation when antiTNF is combined with immunosuppressive agents: a systematic review of randomized controlled trials. Ann Med 2014;46:547-54.

40 Kroetsch JT, Levy AS, Zhang H, et al. Constitutive smooth muscle tumour necrosis factor regulates microvascular myogenic responsiveness and systemic blood pressure. Nat Commun 2017:8:14805.

41 Hamilton JA, Tak PP. The dynamics of macrophage lineage populations in inflammatory and autoimmune diseases. Arthritis Rheum 2009;60:1210-21.

42 Eyles JL, Hickey MJ, Norman MU, et al. A key role for G-CSF-induced neutrophil production and trafficking during inflammatory arthritis. Blood 2008;112:5193-201.

43 Li J, Hsu H-C, Yang P, et al. Treatment of arthritis by macrophage depletion and immunomodulation: testing an apoptosis-mediated therapy in a humanized death receptor mouse model. Arthritis Rheum 2012;64:1098-109.

44 Efimov GA, Kruglov AA, Khlopchatnikova ZV, et al. Cell-type-restricted anti-cytokine therapy: TNF inhibition from one pathogenic source. Proc Natl Acad Sci U SA 2016;113:3006-11.

45 Kontoyiannis D, Boulougouris G, Manoloukos M, et al. Genetic dissection of the cellular pathways and signaling mechanisms in modeled tumor necrosis factorinduced Crohn's-like inflammatory bowel disease. J Exp Med 2002;196:1563-74.

46 Blüml S, Binder NB, Niederreiter B, et al. Antiinflammatory effects of tumor necrosis factor on hematopoietic cells in a murine model of erosive arthritis. Arthritis Rheum 2010;62:1608-19.

47 McCann FE, Perocheau DP, Ruspi G, et al. Selective tumor necrosis factor receptor I blockade is antiinflammatory and reveals immunoregulatory role of tumor necrosis factor receptor II in collagen-induced arthritis. Arthritis Rheumatol 2014;66:2728-38.

48 Grell M, Douni $\mathrm{E}$, Wajant $\mathrm{H}$, et al. The transmembrane form of tumor necrosis factor is the prime activating ligand of the $80 \mathrm{kDa}$ tumor necrosis factor receptor. Cell 1995:83:793-802.

49 Kruglov AA, Nedospasov SA. [Cytokine neutralization at specific cellular source : a new therapeutic paradigm? German Version]. Z Rheumatol 2017:76:163-5. 